\title{
Immunoglobulin Restores Immune Responses to BTH1677 in Patients With Low Levels of Antibodies to Beta-glucan
}

\author{
PETER J. HURLEY ${ }^{1}$, NANDITA BOSE ${ }^{2}$, GAUTAM JHA ${ }^{3}$, MICHELE GARGANO ${ }^{2}$, NADINE OTTOSON ${ }^{2}$, \\ KEITH GORDEN $^{2}$, BLAINE RATHMANN ${ }^{2}$, BEN HARRISON $^{2}$, XIAOHONG QIU $^{2}$ and ARKADIUSZ Z. DUDEK AR $^{1,3}$ \\ ${ }^{1}$ Regions Cancer Care Center, HealthPartners, St. Paul, MN, U.S.A.; \\ ${ }^{2}$ Biothera Pharmaceuticals, Inc., Eagan, MN, U.S.A.; \\ ${ }^{3}$ University of Minnesota, Minneapolis, MN, U.S.A.
}

\begin{abstract}
Background: BTH1677 is a beta-glucan pathogenassociated molecular pattern (PAMP) being evaluated as a novel immunotherapy of cancer. We previously described that the presence of antibodies against beta-glucan $(A B A)$ in serum is necessary for BTH1677 antitumoral activity. We hypothesized that infusion of immunoglobulin can reinstate responses to BTH1677 in individuals with low ABA levels. Patients and Methods: We report two single-patient studies: one in a patient with metastatic colorectal cancer who received BTH1677, combined with tumor targeting antibody cetuximab; and a second in a patient with metastatic neuroendocrine tumor who received BTH1677 combined with immune checkpoint inhibitor pembrolizumab. Results: The patients had low serum titers of $A B A$ and low innate immune effector functionality induced by BTH1677. Addition of intravenous immunoglobulins restored innate immune activity of BTH1677 and induced clinically meaningful anti-tumoral activity, with long-term disease control. Conclusion: Infusion of immunoglobulin can restore activity of BTH1677 in individuals with low serum ABA level.
\end{abstract}

BTH1677, a yeast-derived soluble $\beta$-1,3/1,6-glucan [Imprime PGG; $\beta(1,6)$-[poly-(1,3)-D-glucopyranosyl]-poly- $\beta$-(1,3)-Dglucopyranose) pathogen-associated molecular pattern (PAMP) is a dectin-1 agonist that is being evaluated as a novel cancer therapy in combination with tumor-targeting, anti-angiogenic and immune checkpoint inhibitor antibodies. In phase II randomized clinical studies, BTH1677 has shown promising

This article is freely accessible online.

Correspondence to: Arkadiusz Z. Dudek, MD, Ph.D., HealthPartners Regions Cancer Care Center, Suite 1161 Mail Stop 11101G, 640 Jackson Street, St. Paul, MN 55101, U.S.A. E-mail: Arkadiusz.Z.Dudek@healthpartners.com

Key Words: BTH1677, Imprime PGG, anti-beta glucan antibody, immunoglobulin, immunotherapy. potential to enhance the efficacy of tumor targeting antibodies (cetuximab and rituximab) in metastatic colorectal cancer, nonsmall cell lung cancer (NSCLC), chronic lymphocytic leukemia, non-Hodgkin lymphoma, anti-angiogenics (bevacizumab) in patients with NSCLC (1-5). An encouraging trend of enhanced overall survival with the combination of BTH1677 and programmed cell death protein 1 (PD-1) inhibitor pembrolizumab has also been reported in an ongoing phase II trial in patients with triple-negative breast cancer (6). Mechanistic investigations exploring these different therapeutic combinations in pre-clinical tumor models have shown that BTH1677, being a PAMP, primes the innate immune effector functions to ultimately inspire an adaptive immune responsebased anticancer immunity cycle. BTH1677 enhances the antitumor immune effector functions of myeloid cells, specifically, stimulating antibody-dependent cellular cytotoxicity and antibody-dependent cellular phagocytosis functions of neutrophils and macrophages respectively, reprogramming the immunosuppressive cells in the microenvironment including tumor-associated M2-macrophages and myeloid-derived suppressor cells, and enhancing the antigen presentation capabilities of dendritic cells (7-12). Recently, BTH1677 was also shown to enhance the antibodydependent cellular cytotoxicity mechanism of natural killer cells, mainly via cross-talk with myeloid cells (13).

In order to exert its pleiotropic effects on myeloid cells, BTH1677 needs to form a tripartite immune complex comprising BTH1677, naturally occurring antibodies to betaglucan (ABA) and cleaved by factor 1 complement protein $\mathrm{C} 3 \mathrm{~b}$ (iC3b) opsonin in individuals with a certain threshold level of ABA $(12,14)$. Ex vivo human and in vivo mouse studies have shown that the innate $\mathrm{Fc}$ portion of immunoglobulin $\mathrm{G}$ receptor II A (FcgRIIa), and the pattern recognition receptors complement receptor 3 and dectin-1 are critical for innate immune functional responses of BTH1677 $(11,12,14)$. Innate immune cells from healthy individual with an insufficient ABA level neither bound nor functionally responded to BTH1677 unless rescued by the addition of 
purified ABA or commercial intravenous immunoglobulin $G$ (IVIG) (12). Results from a phase I healthy volunteer trial confirmed that those with an ABA level above a threshold showed immunopharmacodynamic responses to BTH1677, including complement activation and cytokine production, while minimal immune activation was observed in those with a low ABA level (15). Furthermore, retrospective analyses of a randomized phase II clinical trial in which patients with NSCLC were treated with BTH1677 in combination with cetuximab, carboplatin and paclitaxel showed that while overall survival was not different among all study patients, the survival hazard ratio by Kaplan-Meier analysis for those treated with the combination was 0.48 for immunoglobulin $\mathrm{G}$ ABA-positive versus -negative patients $(p=0.03)$ (3).

Encouraged by these findings, we hypothesized that BTH1677 could be administered along with IVIG in order to replenish the ABA concentration to a threshold sufficient to induce critical immunopharmacodynamic responses in patients with cancer with a low ABA level. Herein, we provide clinical evidence based on two single-use, investigator-initiated Investigational New Drug application (IND) clinical cases that IVIG is sufficient to boost the serum ABA level of individuals with low pre-treatment ABA concentrations, and thereby induce BTH1677-dependent immunopharmacodynamic responses that may generate clinically significant outcomes.

Both single-patient IND studies were approved by either Human Subject Protection Committee of University of Minnesota ("Compassionate Use BTH1677 with Cetuximab for a Patient with Metastatic Colorectal Cancer", Institutional Review Board (IRB) Code Number: 1202M10289, IND\#114684) (case 1), or by Regions Hospital Human Subject Protection Committee ("A Pilot Study of Anti-PD-1 Antibody Pembrolizumab and Imprime PGG for Patients with Metastatic Solid Tumor Stable or Progressing on Immune Checkpoint Inhibitor Therapy") IRB Code Number: A17-330, IND\# 137112) (case 2). Patients gave their written informed consent to participate in these compassionate-use studies.

\section{Patients and Methods}

$A B A \operatorname{Ig} G$. The level of IgG ABA in plasma or serum was measured by enzyme-linked immunosorbent assay (Biothera Pharmaceuticals Inc., Eagan, MN, USA) as previously described (12). Briefly, samples were diluted and incubated on a solid phase coated with BTH1677 before washing and incubation with an anti-human IgG secondary antibody labeled with horseradish peroxidase. After washing again, the plate was developed with a 3,3',5,5'tetramethylbenzidine substrate and the amount of IgG ABA determined by comparison to a serum standard.

Soluble terminal complement complex C5b-9 (SC5b-9) complement activation assay. Serum and plasma samples were thawed on ice and the presence of SC5b-9 was quantitated by MicroVue SC5b-9 Plus enzyme immunoassay kit according to the manufacturer's instructions (Quidel, San Diego, CA, USA).

Chemokines and cytokines. All or a subset of cytokines and chemokines including eotaxin, granulocyte-macrophage colonystimulating factor, interferon gamma (IFN $\gamma$ ), IFN $\alpha 1$, interleukin 1 beta (IL1 $\beta$ ), IL1 receptor antagonist, IL4, IL5, IL6, IL7, IL8, IL10, IL12, IL13, IL15, IL17, IL2, IL2 receptor, C-X-C motif chemokine ligand 9 (CXCL9), CXCL10, C-C motif chemokine ligand 2 (CCL2), CCL3, CCL4, CCL5 (RANTES), IFN-inducible T-cell alpha chemoattractant and tumor necrosis factor $\alpha$ were tested using multiplex or single-plex Luminex kits (EMD Millipore, Billerica, MA, USA; and ThermoFisher Scientific, Pittsburg, PA, USA) at various time points before and after BTH1677 infusion. Results were obtained on Luminex xMAP 200 (ThermoFisher Scientific), using xPONENT software and analyzed on Milliplex Analyst software.

\section{Case 1}

An otherwise healthy 54-year-old woman presented with bowel obstruction in 2006. The patients underwent left hemicolectomy which revealed colorectal adenocarcinoma which was metastatic to six out of the eight lymph nodes removed. She was offered adjuvant therapy for her locally advanced disease, but she declined. One year later computed tomography of the abdomen and pelvis revealed enlarged para-aortic lymph nodes. The patient was started on a chemotherapy regimen of FOLFOX with bevacizumab for recurrent disease but was only able to tolerate three cycles. During subsequent follow-up she noted abdominal pain, which correlated with disease progression in a retroperitoneal lymph node. Single-agent bevacizumab was restarted and resulted in improvement of pain. Subsequently, bevacizumab was given intermittently for worsening abdominal pain with improvement in symptoms, however, in November 2011, intolerable and persistent pain developed despite bevacizumab infusions. Positron-emission tomography computed tomography revealed a $2.2 \times 1.8 \mathrm{~cm}$ left para-aortic lymph node with increased metabolic activity. Consequently, she underwent open resection of the lymph node in January 2012. It was a difficult procedure with significant blood loss needing transfusions as the lymph node was adherent to the left renal artery. Histopathology from this resection confirmed lymph node involvement of colorectal adenocarcinoma with extracapsular extension. The tumor did not harbor Kirsten ras oncogene homolog (KRAS) mutation. The resection did not help improve abdominal pain. Computed tomography in February 2012 demonstrated rapid re-growth of tumor with a conglomerate of lymph nodes in the retro-peritoneum with increased metabolic activity. The patient was then offered participation in single-patient IND study of cetuximab and BTH1677. She received standard dose of cetuximab along with BTH1677 at $4 \mathrm{mg} / \mathrm{kg}$. Initially both infusions were given weekly (Figure 1). Treatment 


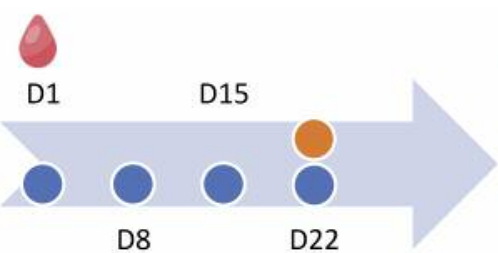

CYCLES 1-5

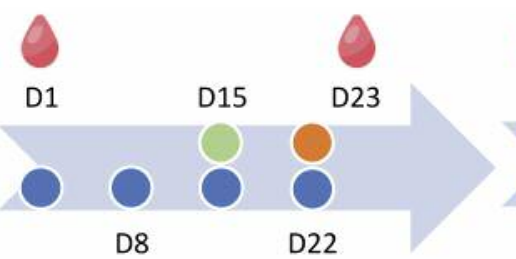

CYCLE 6

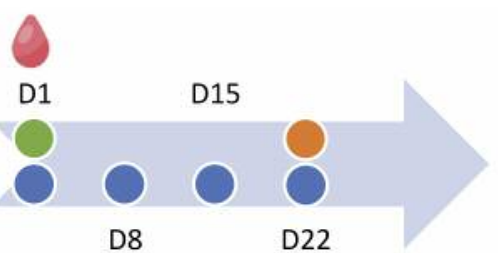

CYCLES 7-11

\section{BTH1677 + CETUXIMAB IVIG $500 \mathrm{mg} / \mathrm{kg}$ \\ BEVACIZUMAB \\ IVIG $1000 \mathrm{mg} / \mathrm{kg}$ \\ BLOOD DRAW (PBMC/ SERUM) \\ (C2-C5, C6, C7-C9)}

Figure 1. Schematic summary of treatment regimen used for compassionate use for case 1. IVIG: Intravenous immunoglobulin; PBMC: peripheral blood mononuclear cells.
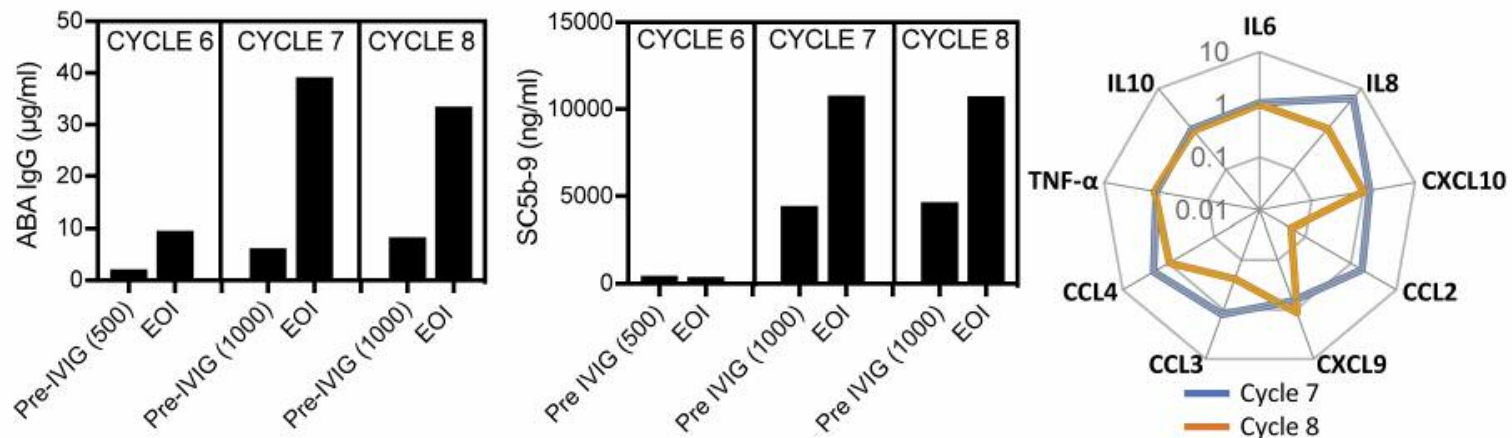

Figure 2. Intravenous immunoglobulin (IVIG) administration prior to BTH1677 resulted in increased immunoglobulin $G$ antibodies against betaglucan (ABA IgG), complement activity and chemokine production. Serum was collected on day 1 prior to infusion, the end of infusion (EOI) or $168 \mathrm{~h}$ post infusion. Left panel: ABA IgG levels were measured by enzyme-linked immunosorbent assay. Center panel: Soluble terminal complement complex C5b-9 (SC5b-9) levels were assessed by solid-phase enzyme immunoassay assay. Right panel: Chemokine levels were measured by Luminex multiplex assay and plotted as fold change in chemokine at the end of infusion relation to the pre-infusion level. IL: Interleukin; CXCL: $C$-X-C motif chemokine ligand; $C C L$ : $C$-C motif chemokine ligand; TNF $\alpha$ : tumor necrosis factor alpha.

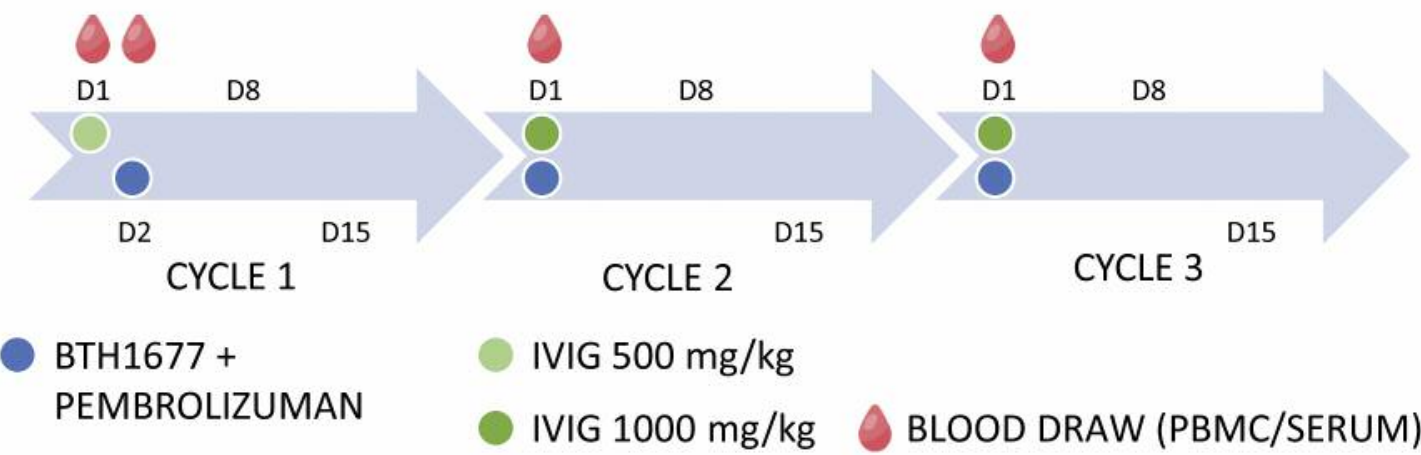

Figure 3. Schematic summary of treatment regimen used for compassionate use for case 2. IVIG: Intravenous immunoglobulin; PBMC: peripheral blood mononuclear cells. 

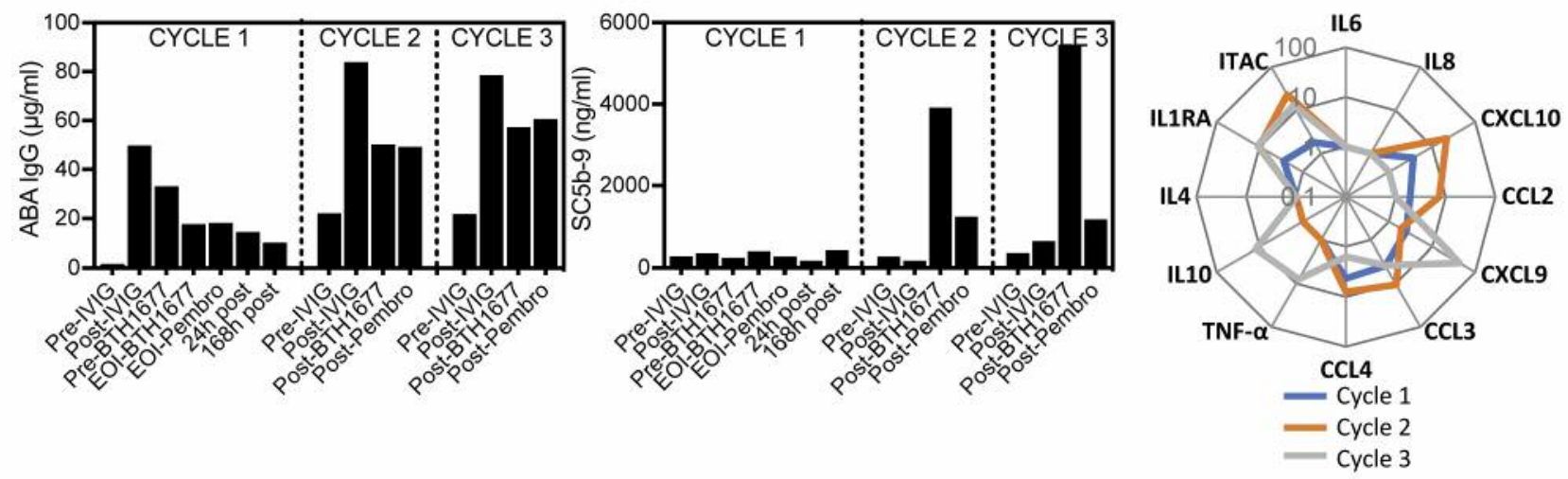

Figure 4. Intravenous immunoglobulin (IVIG) administration prior to BTH1677 resulted in increased immunoglobulin G antibodies against betaglucan (ABA IgG), complement activity and chemokine production. Serum was collected on day 1 prior to infusion, the end of infusion (EOI) or $168 \mathrm{~h}$ post infusion. Left panel: ABA IgG levels were measured by enzyme-linked immunosorbent assay. Center panel: Soluble terminal complement complex C5b-9 (SC5b-9) levels were assessed by solid-phase enzyme immunoassay assay. Right panel: Chemokine levels were measured by Luminex multiplex assay and plotted as fold change in chemokine at the end of infusion relation to the pre-infusion level. IL: Interleukin; IL1RA: IL1 receptor antagonist; CXCL: C-X-C motif chemokine ligand; CCL: C-C motif chemokine ligand; TNF $\alpha$ : tumor necrosis factor alpha; ITAC: IFN-inducible T-cell alpha chemoattractant.

improved abdominal pain so that the patient was able to discontinue narcotic pain medications. She had fatigue, mild acneiform rash, diarrhea, and hypomagnesemia from cetuximab therapy. Radiographically, she had stable disease. In August 2012, after 6 months of treatment, it was realized that she had a low level of ABA $(2.1 \mu \mathrm{g} / \mathrm{ml}$ at cycle 6$)$ and might not be gaining optimal benefit from BTH1677 therapy. It was hypothesized that administering immunoglobulins would increase the ABA level and permit a robust immune response to her cancer. The patients received IVIG at a first dose of $500 \mathrm{mg} / \mathrm{kg}$, and the next dose was increased to 1,000 $\mathrm{mg} / \mathrm{kg}$. Subsequently, she developed a mild infusion reaction and acneiform rash from cetuximab worsened. IVIG infusion was then administered one day prior to cetuximab, with no further episodes of infusion reaction. ABA level and innate immune response were monitored by analysis of pre- and post-treatment serum samples. As shown in the left panel of Figure 2, the infusion of IVIG increased the serum level of ABA. Activation of the complement cascade (determined by the level of C5a, Figure 2, center panel) and cytokine production (Figure 2, right panel) in response to BTH1677 treatment were only achieved at the $1,000 \mathrm{mg} / \mathrm{kg}$ dose of IVIG which elevated the ABA level above $30 \mu \mathrm{g} / \mathrm{ml}$. The patient continued to remain asymptomatic on treatment although there was a mild increase in size of para-aortic lymphadenopathy after 3 months of therapy with cetuximab, BTH1677, and IVIG. Subsequently, she received radiation treatment to the enlarging lymph nodes, at which time all treatment was discontinued. She had a complete radiographic response and remains disease-free after more than 5 years without additional therapy.

\section{Case 2}

An 84-year-old female presented in September 2017 with metastatic islet cell carcinoma whose disease progressed after multiple lines of therapy including octreotide, tyrosine kinase inhibitors (sunitinib and cabozantinib), mammalian target of rapamycin inhibitor (everolimus), and immune checkpoint inhibitor (pembrolizumab). At that time, she was considered for compassionate use of BTH1677 therapy but because her serum baseline ABA level $(1 \mu \mathrm{g} / \mathrm{ml})$ was below the limit thought to be necessary for BTH1677 activation of an immune response, the decision was made to increase serum ABA using IVIG. Subsequently, she enrolled in single-patient IND study with pembrolizumab, BTH1677, and IVIG (Figure 3). Treatment started with IVIG $(500 \mathrm{mg} / \mathrm{kg})$ one day prior to BTH1677 (4 mg/kg), followed by intravenous pembrolizumab (200 mg) once every 21 days. The ABA titer was monitored after each treatment. The initial dose of IVIG $(500 \mathrm{mg} / \mathrm{kg})$ resulted in an increase of ABA level from $1 \mu \mathrm{g} / \mathrm{ml}$ at baseline to $49.9 \mu \mathrm{g} / \mathrm{ml}$ on the day of IVIG infusion. On the next day, the pre-dose ABA level was $33.2 \mu \mathrm{g} / \mathrm{ml}$ that was insufficient to show immune activation (cycle 1; Figure 4). Subsequent doses of IVIG were administered on the same day as BTH1677 and pembrolizumab. The IVIG treatment was increased to $1,000 \mathrm{mg} / \mathrm{kg}$, which increased the patient's ABA level to $89.9 \mu \mathrm{g} / \mathrm{ml}$ (Figure 4, left panel) which then dropped to $17.9 \mu \mathrm{g} / \mathrm{ml}$ post-BTH1677 administration. As shown previously, this drop of free ABA level after BTH1677 infusion is due to complexation with ABA (15). This drop in the ABA level was not observed post pembrolizumab infusion, indicating the specific interaction of $\mathrm{ABA}$ with 
Table I. Size of target lesions in case 2, during therapy and in subsequent treatment.

\begin{tabular}{lccccccc}
\hline & \multicolumn{5}{c}{ Time point } \\
\cline { 2 - 8 } & $\begin{array}{c}3 \text { Months } \\
\text { prior }\end{array}$ & Baseline & $\begin{array}{c}\text { After 2 } \\
\text { cycles }\end{array}$ & $\begin{array}{c}\text { After 4 } \\
\text { cycles }\end{array}$ & $\begin{array}{c}\text { 1 Month after } \\
\text { stopping BTH1677 }\end{array}$ & $\begin{array}{c}\text { 3 Months after } \\
\text { stopping BTH1677 }\end{array}$ & $\begin{array}{c}\text { 2 Months after } \\
\text { starting CAPTEM }\end{array}$ \\
\hline Target lesion size $(\mathrm{mm})$ & 141.2 & 179.9 & 171.1 & 208.6 & 223 & 260 & 237 \\
\hline
\end{tabular}

CAPTEM: Capecitabine and temozolomide.

BTH1677 and not pembrolizumab. Consistent with the finding in case 1, the increase in ABA level pre-BTH1677 dose by the increased dose of IVIG was sufficient to achieve an immune response to BTH1677 $\mathrm{IgG}$ as measured by enhanced complement activity (Figure 4, center panel) and chemokine/cytokine levels (Figure 4, right panel). The patient did not experience any infusion reactions or any side-effects of treatment, with exception of transient aggravation of preexisting myalgias (only during the first cycle). Assessment of disease after two cycles demonstrated a decrease in the size of target lesions in the liver and pancreas by $5 \%$. Imaging after four cycles showed a $21 \%$ increase in size of target lesions from nadir without evidence of new metastatic lesions (Table I). Although correlative parameters suggested immune activation, the decision was made to stop therapy. One month after treatment discontinuation, disease resumed a fast rate of progression similarly to that prior to study initiation. Subsequent therapy with capecitabine and temozolomide regimen, resulted in $9 \%$ reduction in tumors as measured by Response Evaluation Criteria In Solid Tumors version 1.1 (RECIST 1.1) (16) after two cycles, unexpectedly for sixthline therapy, and led to continued control of disease for 10 months. Subsequently disease progressed and the patient died in December 2019.

\section{Discussion}

Our results suggest that IVIG supplementation for individuals with a low serum ABA level can restore BTH1677-mediated immune activation. The minimum dose of IVIG supplementation needed to convert a patient with low endogenous ABA titer into one with an adequate titer to activate an immune response seems to be $1,000 \mathrm{mg} / \mathrm{kg}$. In addition, clinical antitumoral response was seen in both cases with improvement in disease-related symptoms and radiological disease stabilization. Perhaps the most intriguing finding was cancer response to the subsequent therapy (complete response to radiotherapy in the first case, and minor response to chemotherapy in second case) which continued in both patients even after stopping therapy.

This is not surprising as there is precedence of other innate immune modulators, including CD40 agonists, enhancing the antitumor activity of chemotherapeutic agents (17), specifically by modulating the tumor microenvironment. BTH1677, being a PAMP, can prime immune effector functions broadly in addition to eliciting direct antitumor responses through antibody-dependent cellular cytotoxicity and antibodydependent cellular phagocytosis. BTH1677 modifies the tumor microenvironment in several ways. It induces a switch of M2 to M1 macrophages, changes the chemokine milieu, increases infiltration of CD8 T-cells versus regulatory $\mathrm{T}$ cells, and reduces expression of $\mathrm{T}$ cell-suppressive factors such as indoleamine-2,3-dioxygenase and transforming growth factor $\beta$. In addition, BTH1677 enhances antigen presentation capability of tolerogenic dendritic cells, and, most importantly, induces a type-1 interferon signature. All these effects of BTH1677 can collectively induce an inflammatory state systemically such that there is an enhanced response to subsequent therapeutic modalities $(8,9,11,12,18-20)$.

In terms of safety, both patients tolerated therapy very well without added toxicities due to the addition of IVIG (with the exception of infusion reactions).

In summary, this study presented two case studies that demonstrated the ability of IVIG to restore the immunomodulatory ability of BTH1677 in patients with inherently low ABA levels. This finding is critical as clinical activity of BTH1677 may be limited to individuals with a certain threshold level of ABA and the use of IVIG might expand the patient population potentially responsive to BTH1677-based immunotherapy. Planned prospective clinical trials with BTH1677 that will use ABA level as a selection criterion will shed further light on $\mathrm{ABA}$ as a binary or a continuous biomarker. This study highlights the importance of the need for predictive biomarkers in cancer immunotherapy.

\section{Conflicts of Interest}

PJH and GJ declare no conflict of interest; NB, MG, NO, KG, BR, $\mathrm{BH}$, and $\mathrm{XQ}$ are employees of Biothera Pharmaceuticals, Inc, and AZD has stocks in Biothera Pharmaceuticals, Inc.

\section{Authors' Contributions}

AZD, PJH, GJ, NB, and MG designed the study. PJH, and GJ acquired data. $\mathrm{AZD}, \mathrm{NB}, \mathrm{MG}, \mathrm{NO}, \mathrm{KG}, \mathrm{BR}, \mathrm{BH}$, and $\mathrm{XQ}$ provided 
data analysis. PJH, AZD, GJ, and NB prepared the initial draft of the article. All Authors contributed to subsequent article drafts and agreed with the submission of the article for publication.

\section{Acknowledgements}

The Authors would like to thank James P. Zacny, Ph.D. for assistance in preparing the article for submission.

\section{References}

1 Segal NH, Gada P, Senzer N, Gargano MA, Patchen ML and Saltz LB: A phase II efficacy and safety, open-label, multicenter study of Imprime PGG injection in combination with cetuximab in patients with stage IV KRAS-mutant colorectal cancer. Clin Colorectal Cancer 15: 222-227, 2016. PMID: 26975418. DOI: 10.1016/j.clcc.2016.02.013

2 Jacobson CA, Redd R, Reynolds C, Fields M, Armand P, Fisher DC, Jacobsen ED, LaCasce AS, Bose $\mathrm{N}$, Ottoson $\mathrm{N}$ and Freedman A: A phase 2 clinical trial of rituximab and $\beta$-glucan PGG in relapsed/refractory indolent B-cell non-Hodgkin lymphoma. Hematol Oncol 37(S2 Suppl): 521-523, 2019. DOI: 10.1002/hon.207_2631

3 Thomas M, Sadjadian P, Kollmeier J, Lowe J, Mattson P, Trout JR, Gargano M, Patchen ML, Walsh R, Beliveau M, Marier JF, Bose N, Gorden K and Schneller F 3rd: A randomized, openlabel, multicenter, phase II study evaluating the efficacy and safety of BTH1677 (1,3-1,6 beta glucan; Imprime PGG) in combination with cetuximab and chemotherapy in patients with advanced non-small cell lung cancer. Invest New Drugs 35: 345358, 2017. PMID: 28303530. DOI: 10.1007/s10637-017-0450-3

4 Engel-Riedel W, Lowe J, Mattson P, Richard Trout J, Huhn RD, M. Gargano M, Patchen ML, Walsh R, Trinh MM, Dupuis M and Schneller F: A randomized, controlled trial evaluating the efficacy and safety of BTH1677 in combination with bevacizumab, carboplatin, and paclitaxel in first-line treatment of advanced nonsmall cell lung cancer. J Immunother Cancer 6: 16, 2018. PMID: 29486797. DOI: $10.1186 / \mathrm{s} 40425-018-0324-\mathrm{z}$

5 Zent CS, Call TG, Bowen DA, Conte MJ, LaPlant BR, Witzig TE, Ansell SM and Weiner GJ: Early treatment of high risk chronic lymphocytic leukemia with alemtuzumab, rituximab and poly-(1-6)-beta-glucotriosyl-(1-3)-beta-glucopyranose betaglucan is well tolerated and achieves high complete remission rates. Leuk Lymphoma 56: 2373-2378, 2015. PMID: 25676035. DOI: $10.3109 / 10428194.2015 .1016932$

6 O'Day S, Borges VF, Chmielowski B, Rao RD, Abu-Khalaf MM, Stopeck A, Nikolinakos P, Telli ML, Xie B, Shaheen MF, Mattson P, Gargano MA, Cox J, Karantza V, Chisamore MJ, Osterwalder B, Bose N, Uhlik MT and Graff J: An open label, multicenter phase II study combining imprime PGG (PGG) with pembrolizumab $(\mathrm{P})$ in previously treated metastatic triplenegative breast cancer (mTNBC). J Clin Oncol 37, suppl; abstr 2550, 2019. DOI: 10.1200/JCO.2019.37.15_suppl.2550

7 Leonardo SM, Fulton RB, Gorden KB, Fraser K, Harrison B, Kangas T, Jonas A, Yokoyama Y, Ottoson N, Bose N and Graff JR: Imprime PGG, a $\beta$-glucan PAMP (pathogen-associated molecular pattern) activates the direct killing functions of innate immune cells in concert with tumor targeting antibodies. Cancer Res 76(14 Suppl): 80, 2016. DOI: 10.1158/1538-7445.AM2016-LB-080
8 Jonas AB, Chan ASH, Ottoson NR, Qiu X, Fraser K, Kangas TO, Fulton R, Rathmann B, Graff JR and Bose N: Imprime PGG drives adaptive immune resistance within the tumor microenvironment by modulating the myeloid compartment and enhances the efficacy of anti-PD1 antibody in vivo. Cancer Res 76(14 Suppl): LB-087, 2016. DOI: 10.1158/1538-7445.AM2016LB-087

9 Fraser K, Ottoson N, Qiu X, Chan ASH, Jonas A, Kangas T, Graff $\mathrm{J}$ and Bose N: Imprime PGG synergizes with anti-angiogenic antibodies to repolarize the immune microenvironment, suppressing xenograft tumor growth in vivo. Cancer Res 76(14 Suppl): 3280, 2016. DOI: 10.1158/1538-7445.AM2016-3280

10 Fraser K, Chan ASH, Fulton R, Leonardo S, Jonas AB, Qiu X,Ottoson N, Kangas T, Gordon K, Graff J and Bose N: Imprime PGG triggers PD-L1 expression on tumor and myeloid cells and prevents tumor establishment in combination with aPD-L1 treatment in vivo. Cancer Res 76(14 Suppl): 2335 , 2016. DOI: 10.1158/1538-7445.AM2016-2335

11 Fulton RB, Leonardo SM, Jonas AB, Fraser KA, Chan ASH, Ottoson NR, Danielson ME, Bose N, Graff JR and Gorden K: Imprime PGG, a $\beta$-glucan PAMP (pathogen-associated molecular pattern), effectively elicits in vivo maturation of antigen-presenting cells in mice and humans, suggesting potential synergy with checkpoint inhibitor therapy. Cancer Res 76(14 Suppl): LB-089, 2016. DOI: 10.1158/1538-7445.AM2016-LB-089

12 Chan ASH, Jonas AB, Qiu X, Ottoson NR, Walsh RM, Gorden $\mathrm{KB}$, Harrison B, Maimonis PJ, Leonardo SM, Ertelt KE, Danielson ME, Michel KS, Nelson M, Graff JR, Patchen ML and Bose N: Imprime PGG-mediated anticancer immune activation requires immune complex formation. Plos One 11: e0165909, 2016. PMID: 27812183. DOI: 10.1371/journal.pone.0165909

13 Fraser KA, Kangas T, Fulton RB, Leonardo SM, Harrison B, Yokoyama Y, Bose N, Graff JR, Uhlik M and Gorden KB: Imprime PGG, a soluble yeast $\beta$-glucan PAMP, enhancement of anti-tumor responses in combination with tumor targeting antibody is highly dependent on NK cell killing. Cancer Res 78(13 Suppl): 3767, 2018. DOI: 10.1158/1538-7445.AM20183767

14 Bose N, Chan AS, Guerrero F, Maristany CM, Qiu X, Walsh RM, Ertelt KE, Jonas AB, Gorden KB, Dudney CM, Wurst LR, Danielson ME, Elmasry N, Magee AS, Patchen ML and Vasilakos JP: Binding of soluble yeast beta-glucan to human neutrophils and monocytes is complement-dependent. Front Immunol 4: 230, 2013. PMID: 23964276. DOI: 10.3389/fimmu.2013.00230

15 Bose N, Ottoson NR, Qiu X, Harrison B, Lowe JR, Uhlik MT, Rathmann BT, Kangas TO, Jordan LR, Ertelt KE, Jonas AB, Walsh RM, Chan ASH, Fulton RB, Leonardo SM, Fraser KA, Gorden KB, Matson MA, Graff JR and Huhn RD: Immune pharmacodynamic responses of the novel cancer immunotherapeutic Imprime PGG in healthy volunteers. J Immunol 202: 2945-2956, 2019. PMID: 30988115. DOI: $10.4049 /$ jimmunol.1801533

16 Eisenhauer EA, Therasse P, Bogaerts J, Schwartz LH, Sargent D, Ford R, Dancey J, Arbuck S, Gwyther S, Mooney M, Rubinstein L, Shankar L, Dodd L, Kaplan R, Lacombe D and Verweij J: New response evaluation criteria in solid tumours: Revised RECIST guideline (version 1.1). Eur J Cancer 45(2): 228-247, 2009. PMID: 19097774. DOI: 10.1016/j.ejca.2008.10.026

17 Long KB, Gladney WL, Tooker GM, Graham K, Fraietta JA and Beatty GL: IFNgamma and CCL2 cooperate to redirect tumorinfiltrating monocytes to degrade fibrosis and enhance chemotherapy 
efficacy in pancreatic carcinoma. Cancer Discov 6: 400-413, 2016. PMID: 26896096. DOI: 10.1158/2159-8290.CD-15-1032

18 Uhlik M, Harrison B, Gorden KB, Leonardo SM, Walsh RM, Ertelt KE, Gargano M, Chisamore M, Lowe J, Osterwalder B, Bose $\mathrm{N}$ and Graff $\mathrm{J}$ : Imprime PGG, a soluble yeast $\beta$-glucan PAMP, in combination with pembrolizumab induces infiltration and activation of both innate and adaptive immune cells within tumor sites in melanoma and triple-negative breast cancer (TNBC) patients. Cancer Res 78(13 Suppl): LB-129, 2018. DOI: 10.1158/1538-7445.AM2018-LB-129

19 Qiu X, Chan AS, Jonas AB, Danielson ME, Ottoson NR, Michel KS, Leonardo S, Fulton RB, Fraser KA, Kangas TO, Uhlik M, Graff JR and Bose N: Imprime PGG, a novel phase 2 immunotherapeutic, enhances the anti-tumor activity of checkpoint inhibitors (CPI) and suppresses CPI-induced Indoleamine 2,3dioxygenase (IDO) expression. J Immunother Cancer 5: 86, 2017. DOI: $10.1186 / 2051-1426-2-S 3-P 191$
20 Jonas AB, Chan AS, Qiu X, Fraser KA, Ottoson N, Kangas T, Walsh RM, Leonardo SM, Fulton R, Gorden K, Uhlik M, Graff $\mathrm{J}$ and Bose $\mathrm{N}$ : Imprime PGG modulates immunosuppressive myeloid components of the tumor microenvironment and drives enhanced antitumor efficacy in combination with checkpoint inhibitor therapies. Cancer Res 77(13 Suppl): LB-199, 2017. DOI: 10.1158/1538-7445.AM2017-LB-199

Received December 16, 2019

Revised January 20, 2020

Accepted January 23, 2020 